\title{
DENTAL AND PERIODONTAL HEALTH IN BREAST CANCER WOMEN IN ADJUVANT HORMONE THERAPY
}

\author{
Federica Pezzotti ${ }^{1}$, SILVIA LEONE², Marco Invernizzi ${ }^{2}$, Alessandro de Sire $^{3}$, Mario Migliario ${ }^{4}$, Sabrina Pasqua \\ 1 Azienda Ospedaliero Universitaria Maggiore della Carità \\ 2 University of Eastern Piedmont \\ 3 Universita' degli Studi "Magna Græcia" di Catanzaro \\ 4 Amedeo Avogadro University of Eastern Piedmont
}

Funding: The author(s) received no specific funding for this work.

Potential competing interests: The author(s) declared that no potential competing interests exist.

\section{Abstract}

This cross-sectional study aimed to assess oral hygiene in a wide cohort $(n=122)$ of $B C$ patients, thus showing a possible correlation between bone health, vitamin $\mathrm{D}$ status, and oral health in $\mathrm{BC}$ women undergoing tamoxifen or $\mathrm{Al}$ treatment. Our findings showed that BC women had high prevalence of osteopenia/ osteoporosis, hypovitaminosis D, and a very high prevalence of mild/moderate periodontitis and low oro-dental care. This study might be a starting point for future works investigating the correlation between BC, osteoporosis and oral health to define a patient- oriented multidisciplinary management.

\section{Background:}

Breast cancer $(\mathrm{BC})$ is the most common malignancy and cause of mortality in women, presenting also with several treatment-related disabling complications. ${ }^{[1-4]}$ In this scenario, an increased survival rate of women affected by $B C$ with estrogen receptor-positive (ER) tumors has been observed after the introduction of adjuvant therapies, such as tamoxifen (TAM) and aromatase inhibitors (Als), aimed at reducing the proliferative effects of estrogens on breast tissue in ERpositive BC patients. ${ }^{[5]}$ However, these anti-hormonal pharmacological interventions, promoting bone resorption through aromatase blockade at bone tissue level might lower bone mineral density (BMD) with a resulting pathological condition, defined as cancer treatment-induced bone loss (CTIBL). ${ }^{[6]}$ Moreover, BC women undergoing cancer treatments and antiosteoporotic drugs might experience osteonecrosis of the jaw (ONJ) and periodontal tissue diseases, including gingivitis, and infections. ${ }^{[7]}$

\section{Materials and Methods:}

The aim of this study was to assess the oral health status in patients with previous BC in adjuvant hormone therapy with TAM or with Als (anastrozole, letrozole and exemestane). We recruited post-menopausal BC women referred to the Outpatient Clinic for Oncological Rehabilitation of the Physical Medicine and Rehabilitation Unit of the University Hospital "Maggiore della Carità", Novara, Italy from January to June 2020.

All the participants underwent a specialistic oral health evaluation, including the following outcome measures: the 
Decayed, Missing and Filled Permanent Teeth Index (DMFT), to assess dental caries prevalence as well as dental treatment needs; the Oral Hygiene Index $(\mathrm{OHI})$, for the presence of debris/stain and calculus on the dental elements; the Plaque Control and Record Index (PCR), to assess the presence of plaque on the dental elements; Gingival Bleeding Index (GBI), to evaluate gingival inflammation; the Periodontal Screening and Recording Index (PSR), to assess periodontal status; the Winkel Tongue Coating Index (WTCl), to evaluate the amount of tongue coating.

\section{Results:}

Thus, 122 postmenopausal BC women (mean aged 55.6 \pm 10.4 years) were included in the final analysis. DMFT index had a high average value of $16.07 \pm 7.05$ and similarly, $\mathrm{OHI}$ showed that only $11.5 \%(n=14)$ had good oral hygiene while $35.3 \%$ had a sufficient oral health and $53.2 \%(n=65)$ had an insufficient status. PCR index showed that in $33.6 \%(n=41)$ the plaque was present from $50 \%$ and $75 \%$ of the sites investigated and in $18.0 \%$ ( $n=22)$ of BC women in more than $75 \%$. From PSR index assessment, it emerges that only $2.5 \%(n=3)$ had good oral health while $18.9 \%(n=23)$ had gingivitis, $63.1 \%(n=77)$ had a moderate periodontitis and $15.6 \%(n=19)$ had serious periodontitis.

\section{Conclusion:}

Taken together, our findings showed that BC women had a very high prevalence of mild/moderate periodontitis and low oro-dental care. Oral hygiene assessment might be considered as crucial in BC women and could be implemented and screened in the clinical practice even in other disabling conditions. In this context, rehabilitation and oral health interventions could be included within the quality-of-life interventions for an adequate management of BC survivors.

\section{References:}

1. Invernizzi M, Runza L, De Sire A, Lippi L, Blundo C, Gambini D, Boldorini R, Ferrero S, Fusco N. Integrating augmented reality tools in breast cancer related lymphedema prognostication and diagnosis. J Vis Exp 2020; (156). doi: $10.3791 / 60093$.

2. de Sire A, Losco L, Cigna E, et al. Three-dimensional laser scanning as a reliable and reproducible diagnostic tool in breast cancer related lymphedema rehabilitation: a proof-of-principle study. Eur Rev Med Pharmacol Sci 2020; 24:4476-85.

3. de Sire A, Invernizzi M, Lippi L, Cisari C, Özçakar L, Franchignoni F. Blurred lines between axillary web syndrome and Mondor's disease after breast cancer surgery: A case report. Ann Phys Rehabil Med 2020; 63(4):365-67.

4. Paolucci T, Bernetti A, Paoloni M, et al. Therapeutic alliance in a single versus group rehabilitative setting after breast cancer surgery: psychological profile and performance rehabilitation. Biores Open Access 2019; 8(1):101-10. doi: 10.1089/biores.2019.0011.

5. Rizzoli R, Body JJ, DeCensi A, Reginster JY, Piscitelli P, Brandi ML. Guidance for the prevention of bone loss and fractures in postmenopausal women treated with aromatase inhibitors for breast cancer: an ESCEO position paper. European Society for Clinical and Economical aspects of Osteoporosis and Osteoarthritis (ESCEO). Osteoporos Int 2012; 23(11):2567-76.

6. Migliaccio S, de Sire A, Marocci C, et al. Approach in aromatase inhibitors - induced osteoporosis: results from an Italian multicenter observational study. Clin Cases Miner Bone Metab 2018; 15(3):334-39.

7. Taichman LS, Van Poznak CH, Inglehart MR. Self-reported oral health and quality of life of postmenopausal breast 
cancer survivors on aromatase inhibitors and women without cancer diagnoses: a longitudinal analysis. Support Care Cancer 2016; 24(11):4815-24. 